\title{
Nature of largest cluster size distribution at the percolation threshold
}

\author{
Parongama Sen \\ Department of Physics, University of Calcutta, 92 A.P. C. Road, Calcutta 700009, India. \\ e-mail paro@cucc.ernet.in
}

\begin{abstract}
Two distinct distribution functions $P_{s p}(m)$ and $P_{n s}(m)$ of the scaled largest cluster sizes $m$ are obtained at the percolation threshold by numerical simulations, depending on the condition whether the lattice is actually spanned or not. With $R\left(p_{c}\right)$ the spanning probability, the total distribution of the largest cluster is given by $P_{t o t}(m)=R\left(p_{c}\right) P_{s p}(m)+\left(1-R\left(p_{c}\right)\right) P_{n s}(m)$. The three distributions apparently have similar forms in three and four dimensions while in two dimensions, $P_{\text {tot }}(m)$ does not follow a familiar form. By studying the first and second cumulants of the distribution functions, the different behaviour of $P_{t o t}(m)$ in different dimensions may be quantified.
\end{abstract}

Much has been investigated regarding the distribution of cluster sizes as far as percolating clusters are concerned [1 3. There have been some recent studies on the largest cluster size distribution below criticality and also distribution of smaller clusters at the percolation threshold [4]. The largest cluster size distributions, an example of extreme value statistics, is relevant for several physical phenomena like fracture and breakdown. One can obtain distribution functions for the spanning cluster as well as for smaller clusters though the form of the distribution, especially that of the percolating clusters is not simple. In general, it is a non-Gaussian function.

Let $p$ be the probability that a site is occupied in a lattice. At the percolation threshold $p_{c}$, there may or may not exist a spanning cluster. The spanning probability depends on many factors like the kind of percolation (site or bond), type of lattice, boundary conditions etc. [8]. Spanning will occur with a certain probability less than one, and in the study of spanning or percolating clusters at $p_{c}$, only those cases where the lattice spans are taken into account. On the other hand, for the largest cluster size distribution, the calculations will include all the configurations whether the lattice spans or not. The largest cluster, in fact, enjoys a double role in the sense that it may or may not happen to be the spanning cluster. The probability of the smaller clusters being the spanning cluster is relatively much smaller. Even when the lattice is spanned, the largest cluster may or may not be the spanning one.

The event of the lattice being spanned or not cannot be predicted a-priori in random percolation. However, the consequence of the lattice being spanned or not would directly be reflected by the nature of the distribution of the largest cluster which assumes different roles for the two cases. We find that the two distributions of the scaled mass or size $m$ of the largest cluster, denoted by $P_{s p}(m)$ and $P_{n s}(m)$ for the spanning case and the non-spanning case respectively, are indeed different. The scaled size $m=M / L^{D}$, where the mass of the largest cluster is $M$ in a lattice of linear dimension $L$ and $D$ is the fractal dimension. The latter is related to the exponents of percolation and is same for all clusters when they are ranked [9].7.
The total distribution, which can be independently computed, is actually the weighted sum of the two distributions:

$$
P_{\text {tot }}(m)=R\left(p_{c}\right) P_{s p}(m)+\left(1-R\left(p_{c}\right)\right) P_{n s}(m),
$$

where $R_{p_{c}}$ is the spanning probability at $p_{c}$. We investigate the nature of the distributions for hypercubic lattices in two, three and four dimensions, where the Hoshen-Kopelman algorithm 10 is used. Free boundary condition has been used in all dimensions, except that in two dimensions we have also used helical boundary conditions for comparison. In all cases, spanning has been considered from top to bottom. The largest lattice sizes considered are $L=1600, L=98$ and $L=27$ in two, three and four dimensions respectively with typically $10^{6}$ and $10^{5}$ configurations generated for the smallest and largest lattice sizes.

We first compare the distribution of the largest and the spanning cluster sizes in percolating two-dimensional lattices (Fig. 1) and find that they are numerically indistinguishable almost always except for some cases where the size of the clusters are very small. One can ignore that difference and assume that at least for large values, the spanning cluster and the largest clusters are identical. However, for consistency, we will consider the largest cluster in the spanning case strictly and not the spanning cluster if they are different. This also takes care of the fact that we will avoid the ambiguity arising due to the existence of more than one spanning cluster, which may happen in very few cases.

Figures 2-4 show the three distributions for two to four dimensions with free boundary conditions. For clarity, we have shown the distribution for a single lattice size in three and four dimensions, which represents the scaling distribution. Certain features are clear from the figures: the total distribution in two dimensions is quite different in shape compared to those in three and four dimensions. The distribution when the lattice is not spanning, $P_{n s}$, is much more sharply peaked in the higher dimensions while the width of $P_{s p}$, the distribution for the spanning case, is of the same order in different dimensions. These features will be confirmed quantitatively later on. The forms of the two distributions $P_{s p}$ and $P_{n s}$ are clearly 
different and it should be noted that it is not possible to get a collapse by any trivial scale transformation.

The distribution function for the percolating clusters has been fit to the following forms [1] 3]: a power lawexponential function

$$
f(x)=a x^{b} \exp \left(-c x^{d}\right),
$$

or a double exponential function

$$
f(x)=a \exp \left(-b x^{-c}\right) \exp \left(-d x^{e}\right) .
$$

What is important is the appearance of a number of parameters in both the functional forms and estimates from numerical results may turn messy and involve large errors. The focus of the present study is, however, not to obtain the precise form of the function but to compare the gross features of the distributions. Even without a detailed study, it is obvious that the total distribution $P_{\text {tot }}$ in two dimensions has a form very different from that of the individual distributions. For three or four dimensions, although the distributions for the spanning and non-spanning cases are distinct, the total distribution does not carry any signature that it was generated from these two.

That the behaviour of $P_{t o t}$ in two dimensions is different becomes all the more apparent when one attempts to fit the distributions to familiar forms. We do this without emphasis on the accuracy of the estimated parameters. In two dimensions as well as in three and four, the two distributions $P_{s p}$ and $P_{n s}$ fit quite well to the form (3) with different values of the parameters $a, b, c, d, e$. For example, in three dimensions $c \sim 2.8$ and $e \sim 3.5$ for $P_{s p}$ and $c \sim 2.2$ and $e \sim 1.5$ for $P_{n s}$. The total distribution, however, is of the same form only in three and four dimensions. The values of the exponents e.g., in three dimensions are $c \sim 2.5$ and $e \sim 1.5$ for $P_{\text {tot }}$. Although in general the parameters are different, we notice that among the exponents, the values of $c$ are quite close for the three distributions and $e$ is perhaps same for $P_{t o t}$ and $P_{n s}$. In four dimensions also the values of $c$ are comparable for the three distributions: $c$ lies between 1.9 and 2.0 but the values of $e$ are quite different. In two dimensions, however, both $c$ and $e$ are widely different for $P_{n s}$ and $P_{s p}: c \sim 2.5$ and $\sim 1.45, e \sim 3.0$ and $\sim 10.8$ for the two respectively.

Since the boundary condition plays an important role in percolation problems, we also evaluate the distributions with a different boundary condition, namely, helical boundary conditions, in two dimensions. The results are shown in Fig. 5. Here also, there are two distinct distributions $P_{s p}$ and $P_{n s}$. The cluster sizes will obviously be larger for helical boundary condition (HBC) compared to the free boundary case (FBC). The shift in the probability distributions can be explained by this but what is remarkable is that the total distribution shows a hump on the left side, showing that the form of the total distribution is again not conventional. The distribution, with the hump on the left side has a less pronounced form of the plateau-like region compared to the free boundary case. Such a hump has also been observed for small sizes using a renormalisation group scheme [11] and periodic boundary conditions. Hence one can conclude that in general the total distribution for the largest cluster size is not in a familiar form, the effect being strongest in open boundary case.

In order to understand the difference in the behaviour of the total distribution in different dimensions we note the following points. The reason for the characteristic structure of $P_{t o t}$ in two dimensions must be traced back to the features of the two independent distributions from which $P_{t o t}$ is generated. $P_{t o t}$ typically has a plateau like region (FBC) or a weak two peaked structure (HBC). This could be due to two reasons: either $P_{s p}$ and $P_{n s}$, which are both peaked, have negligible overlap; or the width of the distributions are comparable. As the distributions are normalised, this would imply the heights of the peaks are comparable. The value of $R\left(p_{c}\right)$ should not play any part as it is not particularly different in different dimensions.

The overlap between the distributions are not negligible as one can observe from the figures. In order to compare the behaviour in two, three and four dimensions, we evaluate the ratios $r_{1}=m_{s p}^{(1)} / m_{n s}^{(1)}$ and $r_{2}=m_{s p}^{(2)} / m_{n s}^{(2)}$ where $m^{(r)}$ is the $r$ th cumulant of the distributions. The subscripts on $m$ denote the spanning and non-spanning events as usual. The first measure, if high, will indicate that the peaks of the distribution are far apart as $r_{1}$ is a measure of the mean scaled cluster size and lies close to the peak. The second ratio is roughly a ratio of the widths of the distributions which in turn is an estimate of the ratio of the height of the peaks.

We notice very interesting behaviour of the two ratios defined above and shown in Fig. 6. The first ratio $r_{1}$ varies between 1.6 and 1.7 with some weak dependence on dimensionality. $r_{2}$ on the other hand, shows strong dimensional dependence. The values of $r_{1}$ and $r_{2}$ in two dimensions with $\mathrm{HBC}$ and $\mathrm{FBC}$ indicate that both depend on boundary conditions. In two dimensions, $r_{2}$ is close to 1 , indicating that the peaks of the distributions lie at comparable heights. In higher dimensions, $r_{2}$ increases and differs significantly from unity. The latter may therefore be exclusively responsible for the different behaviour of the total distributions in different dimensions.

The observation that there exist two separate distributions for the largest cluster sizes may not appear very surprising. However, it has not been noted in any previous study although distribution functions for percolation is a much studied problem. Also, usually in percolation, quantities and their distributions have the same qualitative behaviour in dimensions below the upper critical dimension. The spanning cluster distributions for example, in different dimensions, could be fit to the same form [3]. The smaller clusters had distribution functions which had familiar form, very small clusters following a Gaussian 
distribution presumably in two dimensions [7]. This is perhaps the first time a novel feature is seen to be present in two dimensions and absent in higher dimensions as far as the total distribution for largest clusters are concerned. Even if one argues that $P_{s p}$ and $P_{n s}$ should be considered as the more fundamental distributions, rough estimates of the exponents for the distributions for these two showed that in two dimensions, they are different by a much larger margin than in higher dimensions, indicating that the two dimensional case is markedly different regarding distributions of largest cluster sizes. This is an effect independent of boundary conditions although the effect may vary for different cases.

Since the largest and the spanning clusters coincide in most cases, $P_{s p}$ may be regarded as a previously known distribution. Hence $P_{n s}$ is the new distribution obtained from the study. We have made a brief comparison of $P_{n s}$ with the distribution of largest cluster sizes below $p_{c}$, as in both cases the lattice does not span. The cumulative distribution $Q_{c u m}(x)$ of the distribution $Q\left(m_{l}\right)$ of the largest cluster size $m_{l}$ below $p_{c}$ is well-known and has the form 4

$$
Q_{\text {cum }}(x)=Q\left(m_{l}<x\right) \sim \exp \left(-\exp \left(-\lambda_{1} x+\lambda_{2}\right)\right)
$$

such that $\ln \left(-\ln \left(Q_{\text {cum }}(x)\right)\right.$ is a straightline when plotted versus $x$. We recover this behaviour but find that for $P_{n s}$, the behaviour of the corresponding cumulative distribution is much more complicated. Hence one can conclude that $P_{n s}$ is a completely independent distribution and different from any previously known distribution in percolation.

We make one last remark: the existence of two distinct distributions at $p_{c}$ is not a unique feature of the largest cluster only, it is true for any cluster which does not span the lattice. But the total distribution will behave differently depending on the rank of the cluster.

I thank Martin Bazant for the numerous discussions which inspired the present work and also for sending unpublished results. I am also grateful to Dietrich Stauffer and an anonymous referee for comments. The computations were done on a Origin 200 at the Calcutta University Computer Center.

[1] S. Havlin, B. L. Trus, G. H. Weiss and D. Ben-Avraham, J. Phys A 18 L247 (1985). A. U. Neumann and S. Havlin J. Stat. Phys. 52203 (1988) H. Saleur and B. Derrida, J. Physique 461043 (1985).

[2] J.-P. Hovi and A. Aharony, Phys. Rev. E 56172 (1997).

[3] P. Sen, Int.J. Mod. Phys. C 10747 (1999)

[4] P. M. Duxbury and P. L. Leath. J. Phys. A 20 L411 (1987).

[5] M. I. Zeifman and D. Ingman, J. App. Phys. 8876 (1987).

[6] M. Bazant, Phys. Rev E 621660 (2000).

[7] P. Sen, J. Phys. A 321623 (1999).

[8] R. M. Ziff, Phys. Rev. Lett. 692670 (1992).

[9] N. Jan, D. Stauffer and A. Aharony, J. Stat. Phys. 92 325 (1998).

[10] J. Hoshen and R. Kopelman, Phys. Rev. B 143428 (1976).

[11] M. Bazant, unpublished. 


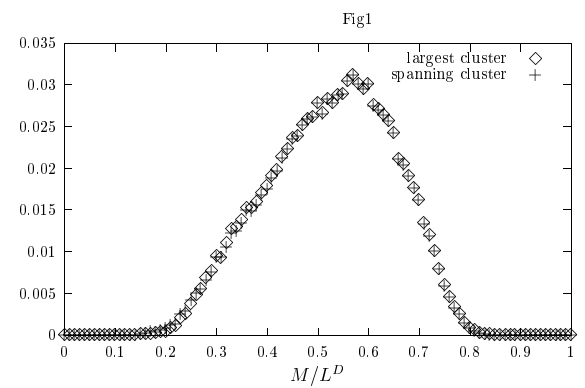

FIG. 1. The comparison of the distributions for the largest cluster and the spanning cluster sizes are shown in two dimensions for $L=600$ with open boundary conditions.

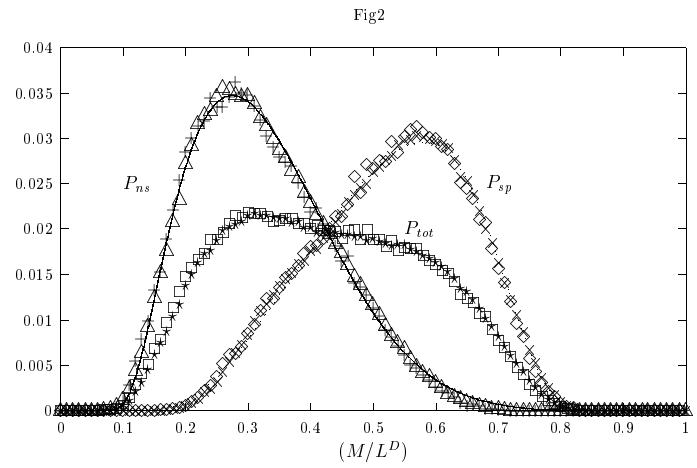

FIG. 2. The three distributions $P_{s p}, P_{n s}$ and $P_{t o t}$ for the scaled mass in two dimensions are shown for two different system sizes $L=400$ (represented by $\times, \triangle$, and $\star$ respectively) and $L=1000$. The best fit lines of the form (3) are also shown for $P_{s p}$ and $P_{n s}$. 


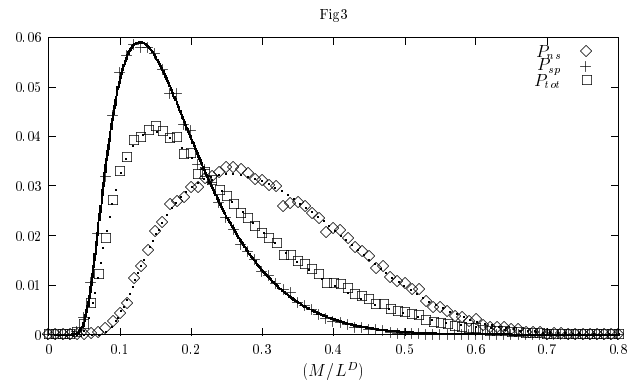

FIG. 3. The three distributions $P_{s p}, P_{n s}$ and $P_{t o t}$ for the scaled mass in three dimensions are shown for $L=60$ along with the best fit lines of the form (3).

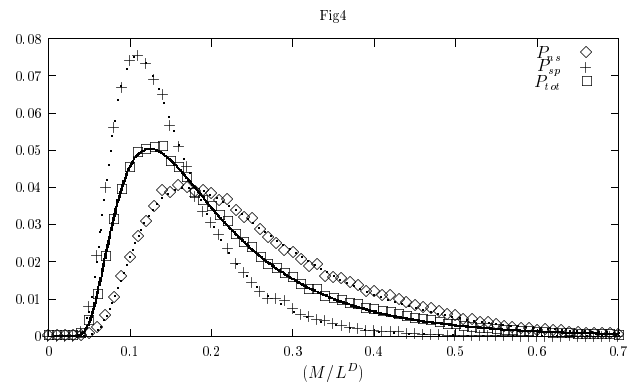

FIG. 4. The three distributions $P_{s p}, P_{n s}$ and $P_{t o t}$ for the scaled mass in four dimensions are shown for $L=21$ alongwith the best fit lines of the form (3). 
Fig 5 Helical

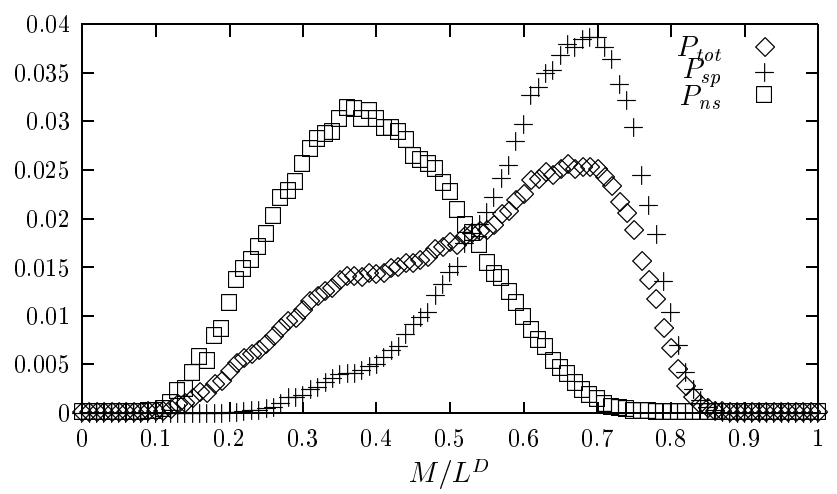

FIG. 5. The three distributions $P_{s p}, P_{n s}$ and $P_{t o t}$ for the scaled mass in two dimensions are shown for $L=600$ with helical boundary conditions. 

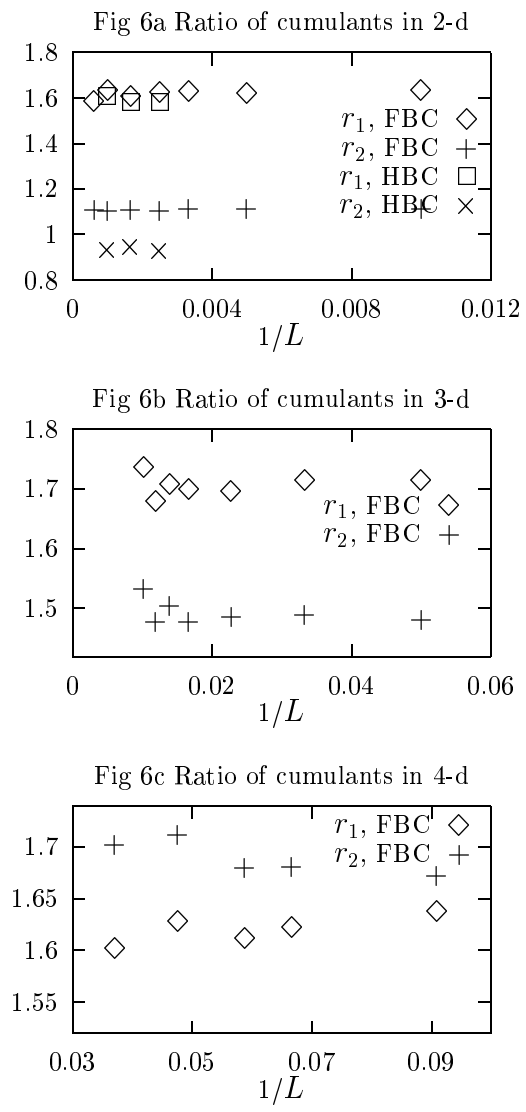

FIG. 6. The ratios $r_{1}$ and $r_{2}$ defined in the text are shown in two, three and four dimensions (FBC and HBC indicate free and helical boundary conditions respectively). 